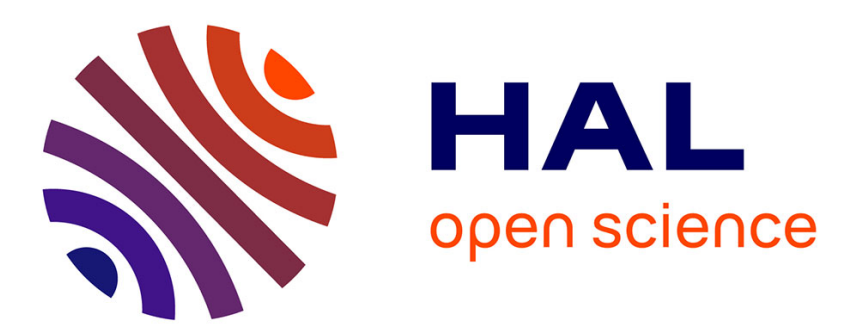

\title{
An innovative subdivision-ICP registration method for tool-path correction applied to deformed aircraft parts machining
}

Richard Béarée, Jean-Yves Dieulot, Patrice Rabate

\section{- To cite this version:}

Richard Béarée, Jean-Yves Dieulot, Patrice Rabate. An innovative subdivision-ICP registration method for tool-path correction applied to deformed aircraft parts machining. International Journal of Advanced Manufacturing Technology, 2010, 53 (5-8), pp.463-471. 10.1007/s00170-010-2875-0 . hal-00939921

\section{HAL Id: hal-00939921 \\ https://hal.science/hal-00939921}

Submitted on 31 Jan 2014

HAL is a multi-disciplinary open access archive for the deposit and dissemination of scientific research documents, whether they are published or not. The documents may come from teaching and research institutions in France or abroad, or from public or private research centers.
L'archive ouverte pluridisciplinaire HAL, est destinée au dépôt et à la diffusion de documents scientifiques de niveau recherche, publiés ou non, émanant des établissements d'enseignement et de recherche français ou étrangers, des laboratoires publics ou privés. 


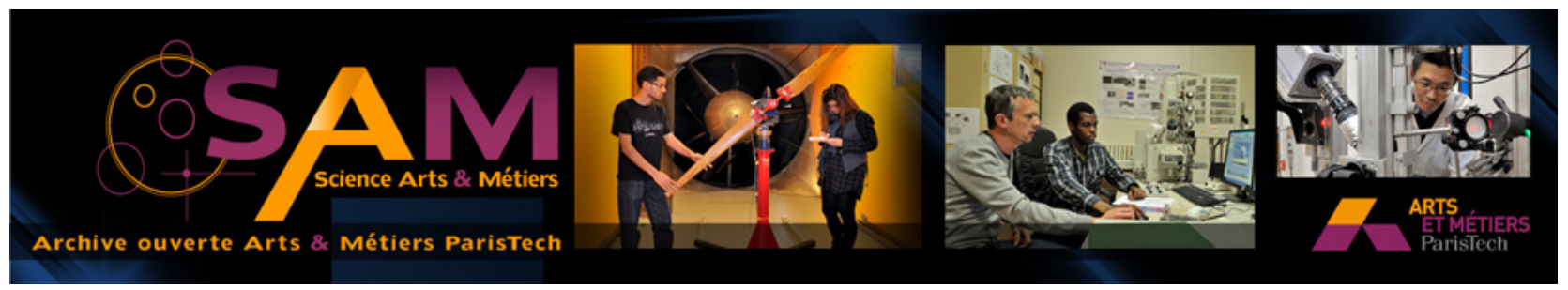

Science Arts \& Métiers (SAM)

is an open access repository that collects the work of Arts et Métiers ParisTech researchers and makes it freely available over the web where possible.

This is an author-deposited version published in: http://sam.ensam.eu

Handle ID: .http://hdl.handle.net/10985/7735

\section{To cite this version :}

Richard BEAREE, Jean-Yves DIEULOT, Patrice RABATE - An innovative subdivision-ICP registration method for tool-path correction applied to deformed aircraft parts machining International Journal of Advanced Manufacturing Technology - Vol. 53, n5-8, p.463-471 - 2010 


\title{
An innovative subdivision-ICP registration method for tool-path correction applied to deformed aircraft parts machining
}

\author{
Richard Béarée • Jean-Yves Dieulot • Patrice Rabaté
}

\begin{abstract}
A new and fast registration algorithm has been proposed to update the tool-path of a deburring robot, intended to machining composite workpieces under gravity and clamping deformations. A Subdivision Iterative Closest Point algorithm, which considers different parts of the contour with respect to curvature, allows to obtain far better results than classical methods, without complicated assumptions or computations. The procedure has shown to be effective for porthole and nose-cone deburring. Experimental tests conducted on robotic milling workcell demonstrated the efficiency of the registration method.
\end{abstract}

Keywords Iterative Closest Point - Image registration $\cdot$ Deburring $\cdot$ Aeronautics • Machining robot

R. Béarée $(\varangle)$

Laboratoire d'Electronique et d'Electrotechnique de Puissance, Arts et Métiers ParisTech, 8 bd Louis XIV, 59800 Lille Cedex, France

e-mail: richard.bearee@ensam.eu

J.-Y. Dieulot

Laboratoire d'Automatique, Génie Informatique et Signal

UMR 8146 CNRS Polytech-Lille, Cité Scientifique,

59650 Villeneuve d'Ascq, France

e-mail: jean-yves.dieulot@polytech-lille.fr

P. Rabaté

EADS Innovation Work, 7, route de Bray, 80300 Méaulte, France

e-mail: patrice.rabate@eads.net

\section{Introduction}

New aircraft designs make a more extensive use of composite materials, which allow to construct large components. However, increasing the size of workpieces requires the use of bigger $\mathrm{CNC}$ machines. As an alternative, 6 d.o.f. robots equipped with a spindle have been considered for deburring operations, allowing higher flexibility and the use of coarse clamping devices [21]. In this case, the workpiece will be roughly positioned, and the trajectory of the robot within space should be registered using the original computer-aided design (CAD) model. However, clamping and gravity forces generate deformations which are not negligible when considering high-dimension composite plates, and should be taken into account in the registration procedure (Fig. 1) As an example, it will be necessary to cut an oval shape into the fuselage to obtain a round porthole once the clamping devices are released. Lengthy re-engineering procedures can be considered which consist of estimating the deformations using finite-element methods and embed those into the CAD/CAM design (see e.g. [19]). However, when considering deburring operations, only the contours of the hollow moulded pieces should be processed, which, combined with small deformation hypotheses, allows on-site registration. Typically, a cloud of CAD model points has to be matched with the measurements given by a 3D measurement device (e.g. advanced CMMs, laser trackers, articulated arms with scanner, handscanner), usable in a manufacturing context.

The Iterative Closest Point (ICP) approach is the most popular for modelling a geometric transformation which allows the matching of a cloud of points with a model [2]. ICP is an iterative descent procedure which 


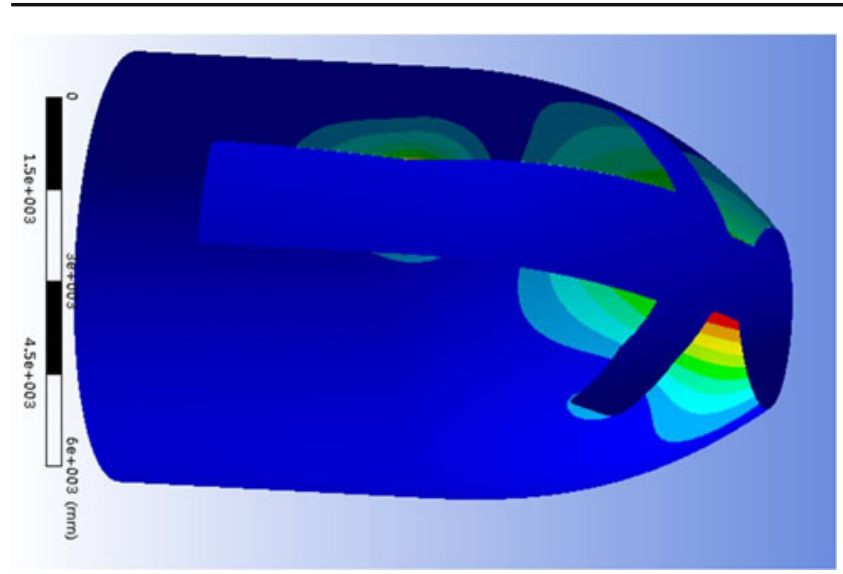

Fig. 1 Deformation of a complex composite part under clamping and gravity forces

aims at minimizing the sum of the squared distances between all points in a dataset (a cloud of points representing a real object) and their closest points in a model. The rigid transformation (find a translation coupled to a rotation) which aligns those sets can be solved in a least squares sense with a closed form solution, and has been shown to be performing for nondeformed images [7,14], with applications in workpiece manufacturing, mainly for CAI purposes after machining $[4,6,16,20]$. Pottmann et al. [17] reveal that the ICP algorithm needs many iterations to converge, but that using local quadratic approximants of the squared distance function allows faster registration. Kim and Kim [11] and Jost and Hügli [9] propose a hierarchical model point selection and a $2 \mathrm{D}$ logarithm data point search which accelerate the ICP convergence, at the price of constructing a K-D tree. Okada and Hebert [15] use a combination of a fast ICP, a two-step ICP, and an estimation of deformation vectors using an accelerated version of the RPM algorithm, splitting the point cloud into deformed and non-deformed parts. In [22], authors use signal-to-noise ratio to improve ICP for inspection of deformed part. No work has been reported for deburring operations, and very few for robot registration [18]. Extensions to deformed images can be handled by introducing additional stiffness terms into the model $[1,10,12,13]$, but the main drawback lies in the expertise needed for working these methods out, and the computational burden which is not always compatible with real-time production.

As, in practice, the workpiece curvature and flexure are rather small (e.g. deburring a hole on a curved plate), it is possible to obtain the deformation of the contour using a projection of the trajectory (once registered using ICP); this deformation can be modelled and then compensated, which avoids the introduction of the aforementioned non-rigid terms. However, when considering more complex structures (e.g. deburring the cockpit into the nose-cone barrel), a simple model is impossible to obtain and a region-based (where regions exhibit small curvature) algorithm can be considered. R-ICP approaches determine generally a unique rigid transformation using a regional weighted Least Square criterion [3], which can also be found when considering images with multiple views [5].

This paper presents a rapid registration procedure applied to contour deburring into a flexible composite workpiece, which uses an ICP method with contour subdivision, denoted as Subdivision-ICP (S-ICP). Each region is registered, and next, smoothing procedures are provided for connecting adjacent regions. Finally, the inverse transforms will be applied to update the robot trajectory.

\section{Registration methods using deformation modelling}

\subsection{Iterative Closest Point and contour matching}

ICP allows to register two points clouds in an iterative way, and to estimate the rigid transformation which aligns them; at each step, pairwise points (in a closest point sense) of the two sets are matched, so that the mean distances between the pairs are minimized.

Let two sets of points, $X=\left\{\vec{X}_{i}\right\}, i=1 \cdots N_{x}$, which represents the model set to be registered and $Y=$ $\left\{\vec{Y}_{i}\right\}, i=1 \cdots N_{y}$ the data set which represents the actual surface. As the number of points can be different, the sets $X$ and $Y$ generally do not match one-toone. In order to match the two datasets, quaternion algebra is introduced [8]. The quaternion denoted $q=$ $\left[q_{0}, q_{1}, q_{2}, q_{3}\right]$ is used to represent the $3 \mathrm{D}$ rotation, $T$ is a translation vector, $R$ is a rotation matrix and $\Phi$ is a function which associates an element $\vec{X}_{i}$ of the set $X$ with its closest neighbor $\vec{Y}_{i}$ in the set $Y$,

$\Phi\left(\vec{X}_{i}\right)=\min \left\|\vec{X}_{i}-\vec{Y}\right\|$.

The $3 \times 3$ rotation matrix generated by a unit quaternion is given by:

$R=\left[\begin{array}{lll}q_{0}^{2}+q_{1}^{2}-q_{2}^{2}-q_{3}^{2} & 2\left(q_{1} q_{2}-q_{0} q_{3}\right) & 2\left(q_{1} q_{3}+q_{0} q_{2}\right) \\ 2\left(q_{1} q_{2}+q_{0} q_{3}\right) & q_{0}^{2}+q_{2}^{2}-q_{1}^{2}-q_{3}^{2} & 2\left(q_{1} q_{3}-q_{0} q_{1}\right) \\ 2\left(q_{1} q_{3}-q_{0} q_{2}\right) & 2\left(q_{2} q_{3}+q_{0} q_{1}\right) & q_{0}^{2}+q_{3}^{2}-q_{1}^{2}-q_{2}^{2}\end{array}\right]$

The ICP algorithm can be split up in four steps which yield the transform $\mathcal{F}=(R, T)$ that minimizes 
the distance between the registered model set $X$ and the actual dataset $Y$ :

a. Initialize $X_{k}$ with $X$, initialize $k=0$

b. For every point $\vec{X}_{i}$ of $X_{k}$, search the closest neighbor $\vec{Y}$ in $Y$.

c. Using the corresponding pairs of points, determine rotation $R_{k}$ and translation $T_{k}$ which minimize the mean quadratic error $J_{k}$ :

$$
J_{k}\left(R_{k}, T_{k}\right)=\frac{1}{N_{X}} \sum_{i=1}^{N_{X}}\left\|R_{k} \vec{X}_{i}+T_{k}-\Phi\left(\vec{X}_{i}\right)\right\|
$$

Several techniques can be used to solve the problem, in our case quaternion algebra allows to obtain the transformation $\mathcal{F}_{k}$.

d. The new set is updated as $X_{k}=R_{k} X_{k-1}+T_{k}$, and step b) is repeated if $J_{k}\left(R_{k}, T_{k}\right) \leq \delta$, where $\delta$ is a predefinite threshold, or if $k \geq k_{\max }$, where $k_{\max }$ is the maximum number of iterations. The final transform is noted $\mathcal{F}=(R, T)$.

The deburring application allows to reduce the number of points to a neighborhood $X=\left\{\vec{X}_{i}\right\}$ of the closed contour $\mathcal{C}$ to be processed (e.g. a porthole) lying on a surface $\mathcal{S}$ (a composite plate). The theoretical surface (model set) is obtained via a CAD package and is originally represented by a set of triangles obtained via Delaunay triangulation. The actual surface can be obtained by many 3D measurement devices (e.g. a hand-scanner moved by an operator or any automated device) in a neighborhood of the contour to be processed. A first common step to the methods which are considered in this paper consists of selecting points (theoretical and real) that belong to the contour. This can be performed by comparing, for every point, the number of neighbors (using the mean distance between points) and the number of triangles which the point belongs to. When these numbers are different, the point belongs to a contour. When considering the actual workpiece, the contours should be validated by the user to eliminate outliers or wrong contours resulting from imprecise, noisy or missed data points. It is well known that the registration precision increases with the sampling density, until some threshold is reached, for which no further appreciable improvement can be noticed [19].

\subsection{A pragmatic determination of the deformation using projection methods}

When considering high-dimension and small curvature workpieces and assuming small deformations, an approximate of the deformation may be obtained using an orthogonal projection of the (coarsely) registered model onto the plane $P$ that approximates the registered model in the Least Square sense. Let $X^{\prime}$ the set registered by the initial ICP algorithm, $\vec{X}_{i}^{\prime}=R \vec{X}_{i}+T$. The orthogonal projection $Q$ of the registered set $X^{\prime}$ onto $P$ is performed as: $\overrightarrow{q_{i}}=Q\left(\vec{X}_{i}^{\prime}\right)$ :

An approximate of the deformation will be

$\vec{d}_{i}=\vec{q}_{i}-\vec{X}_{i}^{\prime}$

If the deformation can be modelled using some function $f($.) - e.g. a spline function-, this model can be embedded into the registration procedure. As an example, the deformation of a composite plate can be very roughly approximated, in a neighborhood, by a parabola of equation $z=\alpha w^{2}$, where $\alpha$ is a parameter to be determined. Of course, such an approximation of the deformation is not exact, since a projection on a plane which approximates the registered surface is performed, but it may be well enough when assuming small deformations. In order to debur the final contour $\mathcal{C}=\left\{\vec{C}_{i}\right)$ (e.g. of the porthole on the fuselage of the aircraft) the following transform should be applied:

$\Gamma / \vec{\gamma}_{i}=f\left(R \vec{C}_{i}+T\right)$

Note that the normals to the workpiece should be computed again so that machining should be performed in a correct way (e.g. orthogonal to the workpiece). The idea of handling a finer registration procedure after a coarse matching has been worked out in CAI problems such as in [16].

Figures 2 and 3 present the results of the registration of a porthole in a composite plate; for the sake of simplicity, only 1,500 theoretical points have been

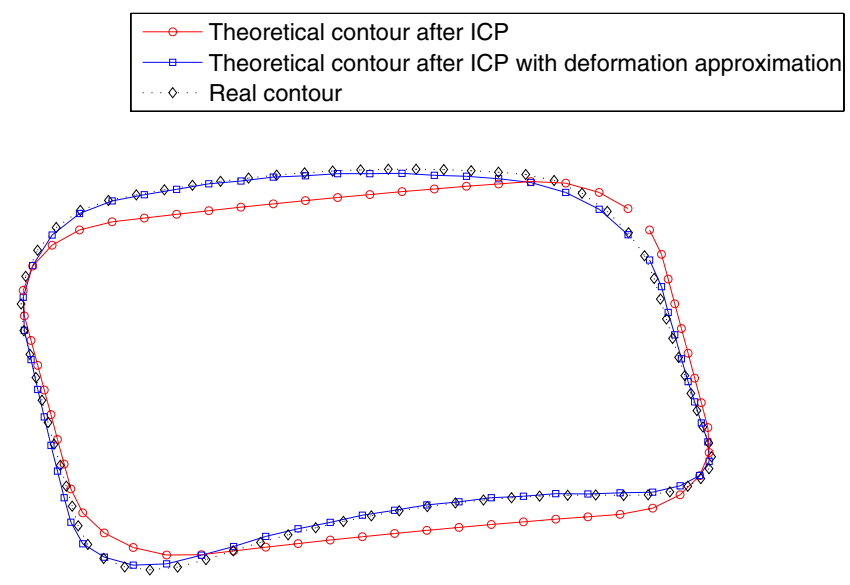

Fig. 2 Deburring a porthole in a double curvature plate: theoretical contour registered on the real deformed workpiece 


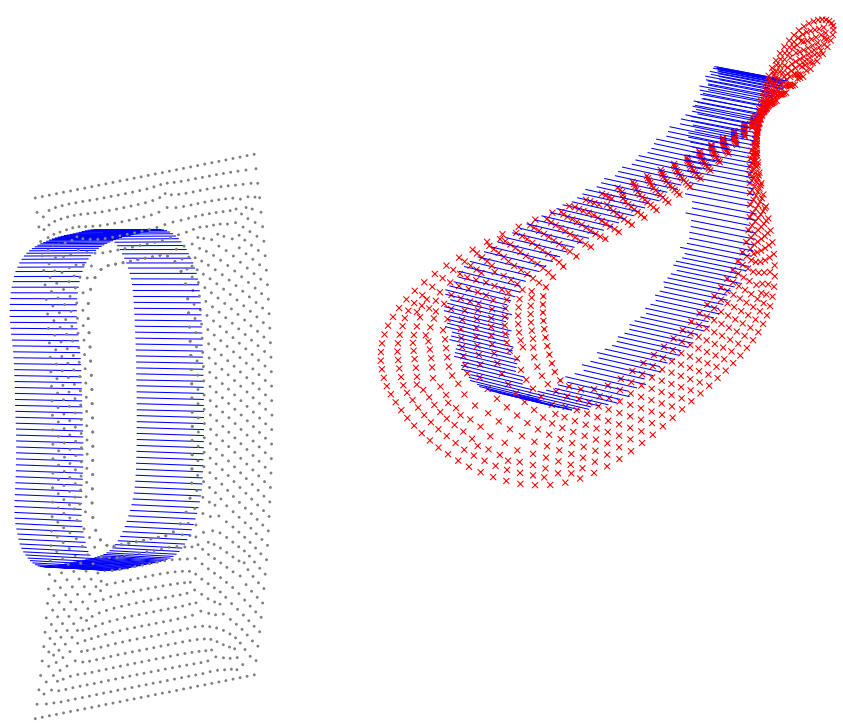

Fig. 3 Deburring a porthole in a double curvature plate: normals of the trajectories on the theoretical workpiece (left) and registered trajectories on the deformed workpiece (right)

considered, which corresponds to a precision of $5 \mathrm{~mm}$. The maximum deformation in a neighborhood of the contour is $11 \mathrm{~mm}$. The mean square error after registration (ICP and deformation compensation) is $3.3 \mathrm{~mm}$, which corresponds to $65 \%$ of the standard error obtained with a rigid ICP.

The precision of registration can be increased when considering a higher number of points and thus finer scanning measurements (typically, the required precision will lie around $0.1 \mathrm{~mm}$ ). Performances are, of course, clearly dependent on the accuracy of the deformation model.

\section{Subdivision method}

\subsection{Contour subdivision}

In the previous method, the deformation was obtained using a projection on a plane which would approximate the contour of the surface to be deburred, and has proven to be successful for such simple surfaces as a porthole. This method is untractable when considering complex workpieces with complicated deformations such as deburring a cockpit into a nose cone (Fig. 4). In this case, it is proposed to divide the surface into quasi-planar surfaces and the contour into a number of quasi-linear curves. An appropriate subdivision method which addresses the problem of overlapping contours and/or connection between registered contours should be designed. The S-ICP algorithm can be split up in the following steps:

a. Detect high curvature contours (with user-defined tuning parameters) and store corresponding points $\left\{\vec{H}_{1}\right\}$.

b. Subdivide low-curvature contours-using subdivision points $\left\{\vec{H}_{2}\right\}$-, for precision purposes.

c. Determine neighboring areas (using user-defined maximum distance to the contour)

d. Add stability points to the set $\mathrm{H}_{2}$

e. Perform rigid ICP, and transform the theoretical contour and the stability points

f. Detect the nearest neighbor in the real image for every registered theoretical point

g. Perform ICP on every sub-contour piece (note that every sub-contour overlaps its neighbors)

h. Perform a smooth weighting procedure for connecting overlapping areas

i. Perform the transformation on the theoretical tooltrajectory (using the nearest neighbor on the theoretical contour)

Many methods can be considered to define the curvature of a point belonging to the contour. The radius of curvature was computed as the radius of the osculating circle (which passes through the point and its nearest neighbors belonging to the contour). Now, a set of subdivision points can be designed such as: $H_{1}=\left\{\overrightarrow{H_{1, i}}\right\}, i=1 \cdots N_{h}$, where

$\overrightarrow{H_{1, i}} \in H / \rho\left(\overrightarrow{H_{1, i}}\right)>\rho_{T}$,

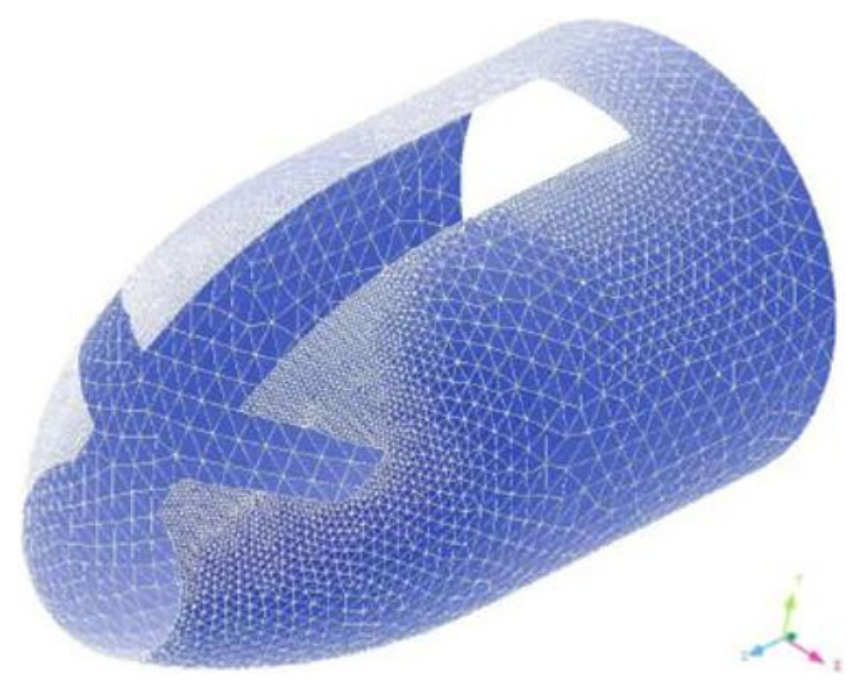

Fig. 4 Deburring a nose-cone (full-barrel) in composite materials 
where $\rho_{T}$ is a threshold curvature radius. For practical purposes, it is possible to divide long contour pieces into small ones, introducing additional subdivision points:

$k \epsilon<s\left(\overrightarrow{H_{2, i}}\right)-s\left(\overrightarrow{H_{2, i-1}}\right)<(k+1) \epsilon, k \in \mathbb{N}$

where $s$ is the curvilinear abscissa and $\epsilon$ is a threshold corresponding to the maximum distance between points, $k$ is the number of points between $\overrightarrow{H_{2, i-1}}$ and $\overrightarrow{H_{2, i}}$. Such a resulting contour is represented in Fig. 5 for the porthole example. The different segments (a number of 6) are located between the markers. Note that all these segments overlap.

\subsection{Scaling ICP registration}

A first ICP procedure is performed on the contour $X$ as before, yielding a registered contour $X^{\prime}$. Thus, the SICP procedure will only refine the results of the coarse ICP registration, and stability and segment connection problems will be easier to handle since only slight modifications will have to be done. Since the initial contour was divided into a set of segments, a new set of registered segments are obtained using the ICP procedure. Now, an ICP algorithm can be performed for each of these new segments (step g.), i.e. every segment of $X^{\prime}$ is registered with respect to the dataset $Y$.

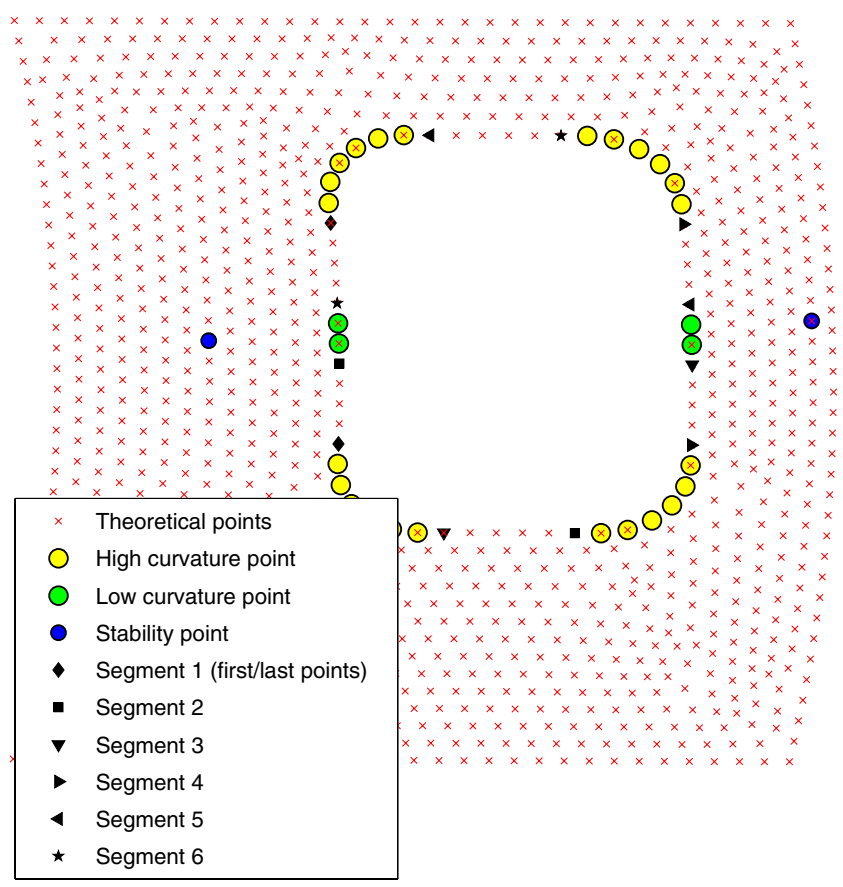

Fig. 5 Subdivision for a porthole
To avoid connection problems, an overlapping range (from $20-40 \%$ ) between segments was allowed; the procedure which handles overlapping part consists of computing a weighted mean of the registered neighboring segments. Let $\overrightarrow{v_{z}}, \overrightarrow{v_{z+1}}$ be the vertices of zones $z, z+1$. Weights were chosen as sinusoidal and the final transformed points $\vec{P}_{i}^{\prime}$ of the overlapping areas will be obtained as:

$H\left(\vec{P}_{i}^{\prime}\right)=\omega_{z}\left(R_{z} \vec{P}_{i}^{\prime}+T_{z}\right)+\omega_{z+1}\left(R_{z+1} \vec{P}_{i}^{\prime}+T_{z+1}\right)$,

where

$\omega_{z}=g\left(\overline{v_{z} p_{i}^{\prime}}, \overline{v_{z+1} p_{i}^{\prime}}\right)$

e.g.

$g\left(\overline{v_{z} p_{i}^{\prime}}, \overline{v_{z+1} p_{i}^{\prime}}\right)=\sin \left(\frac{\pi \overline{v_{z+1} p_{i}^{\prime}}}{2 \overline{v_{z} p_{i}^{\prime}}}\right)$

Note the existence of stability points $\overrightarrow{S P}$ (step d.), chosen in the surface $\mathcal{S}$, outside the contour. $\overrightarrow{S P}^{\prime}$ is the set corresponding to the transformation of $\overrightarrow{S P}$ by the transform $\mathcal{F}=(R, T)$ of the primary ICP, for which the nearest neighbors are searched in $Y$. During the S-ICP procedure, these points are added to every segment of $X^{\prime}$, and their nearest neighbors to $Y$. This allows to obtain realistic rotation matrices and can be considered as a security parameter, in particular with respect to missing data in the scanned image. Their influence will be tackled in a further study.

\section{Results}

\subsection{Practical applications of the registration method}

First results are shown on a simplified workpiece (deburring a porthole with a double curvature). Next, the method was tested on a nose cone for which the deformation under gravity and clamping forces was obtained using a finite element software (ANSYS ${ }^{\mathrm{TM}}$ ).

Figure 6 shows that efficient registration can be obtained for porthole deburring.

For a complex workpiece (Fig. 7), one notes that the stability points, subdivision points, overlapping segments determination procedure are fully automated, one such tuning parameters being defined by the user, resulting into the subdivision in Fig. 8. Results in Fig. 9 underline the relevance of the S-ICP algorithm, which does not require a computation of the deformation 


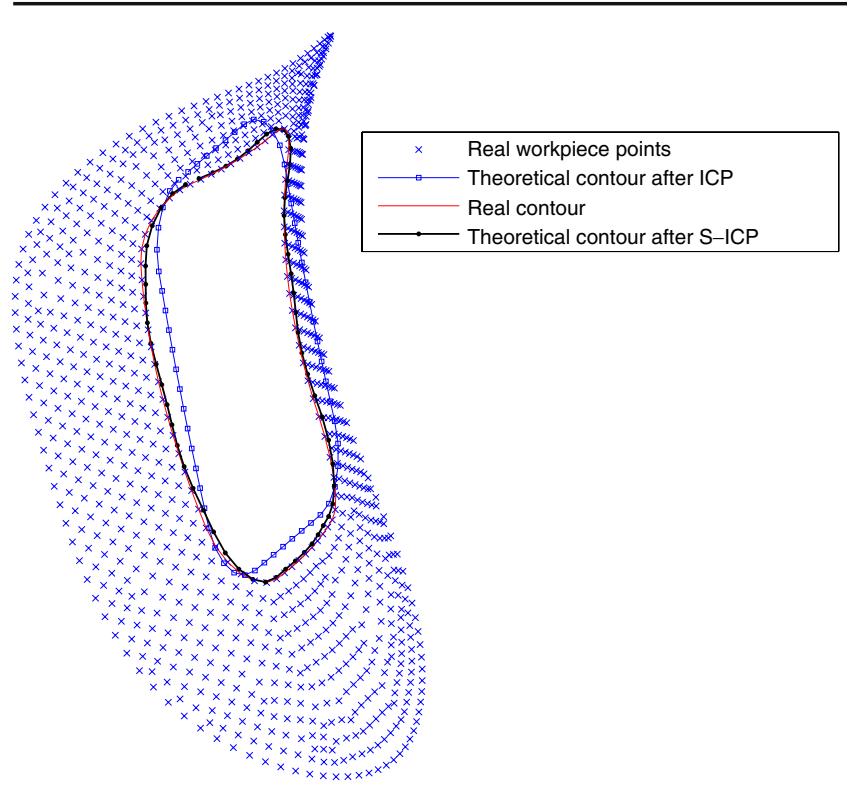

Fig. 6 Registration of a porthole with ICP and S-ICP

(which is quite complex, as suggested by Figs. 2 and 7). The workpiece can be registered quite fast, with the only requirement to obtain a real dataset. This is really one of the main interests of the method, because on-site registration can be performed by a trained operator and avoids further re-engineering. Figure 10 shows results for primary ICP and S-ICP. Note that

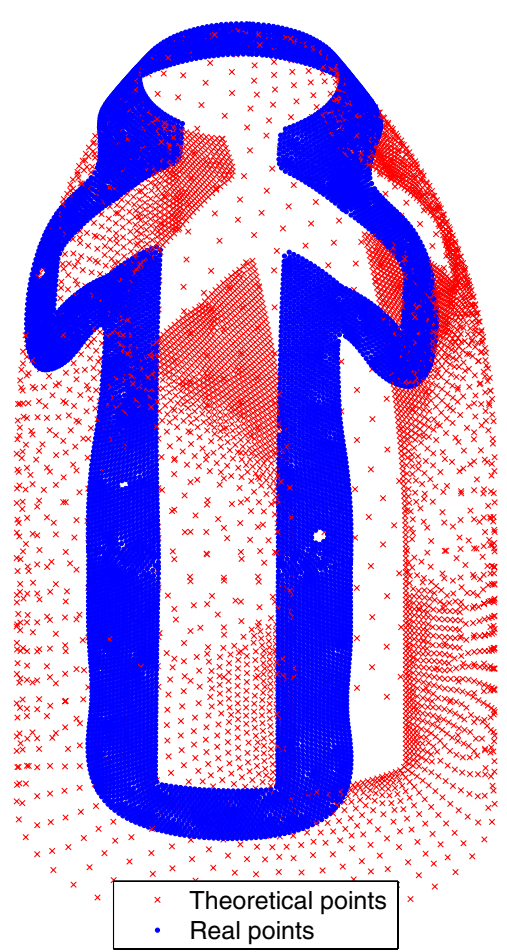

Fig. 7 Nose-cone model and measurements

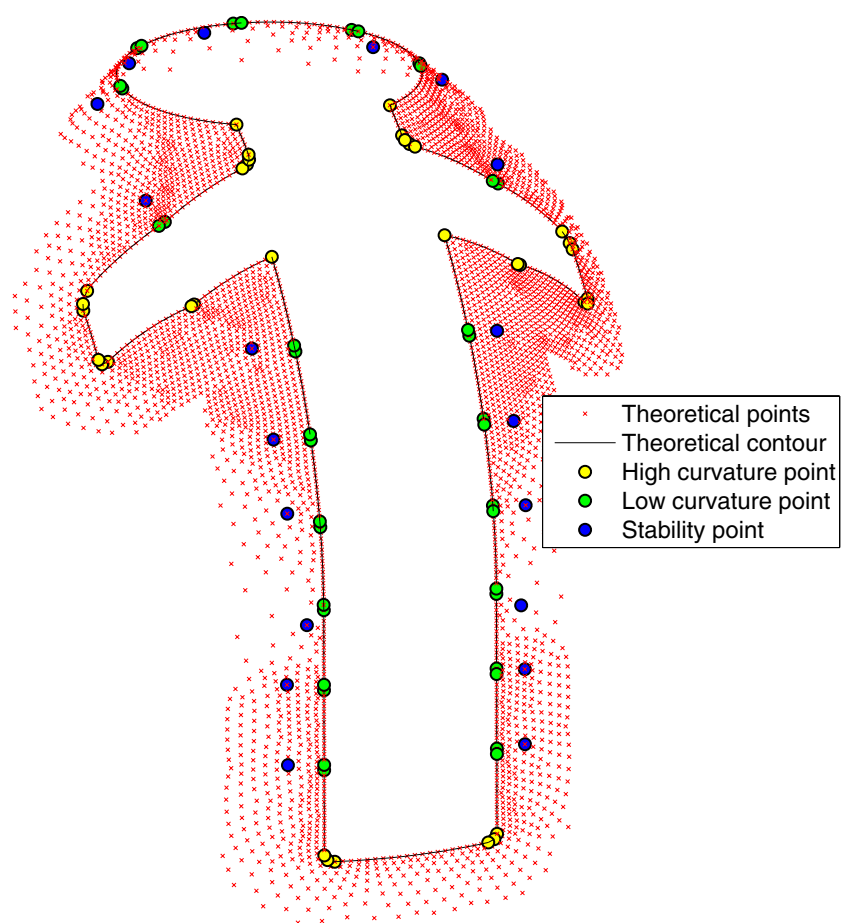

Fig. 8 Subdivision points on a nose-cone

the signature of the deformation resulting from gravity and clamping constraints can be retrieved when using the ICP method. S-ICP yields a mean error of

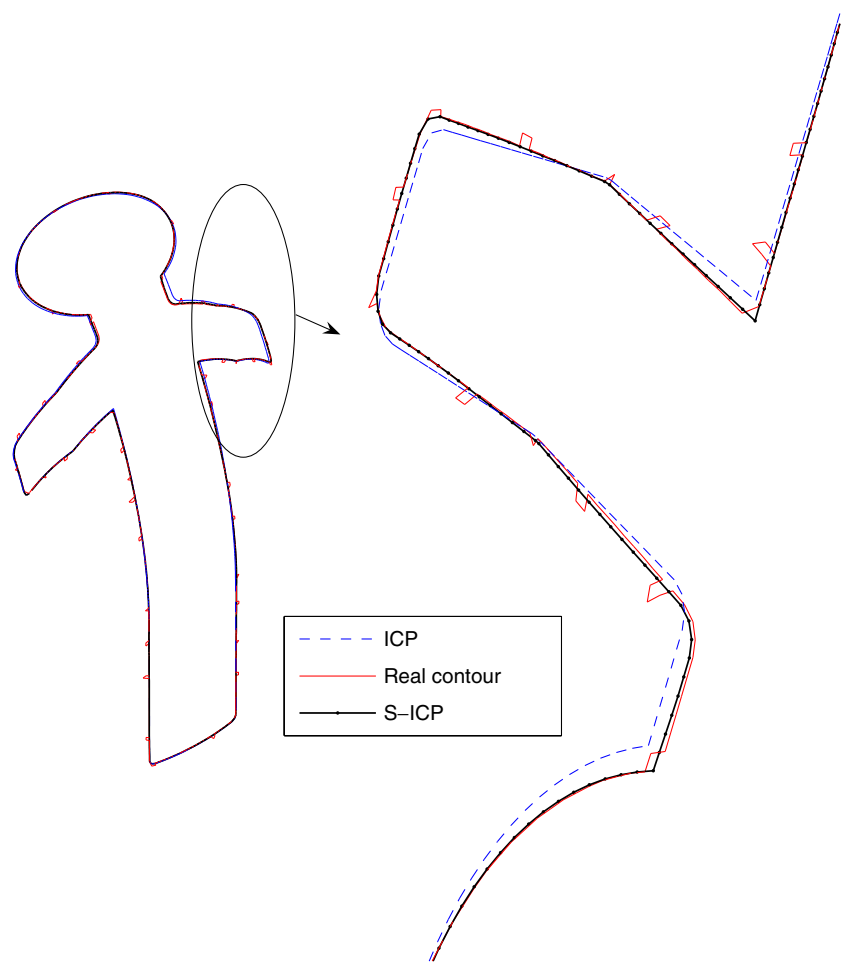

Fig. 9 Cockpit deburring into a nose-cone 


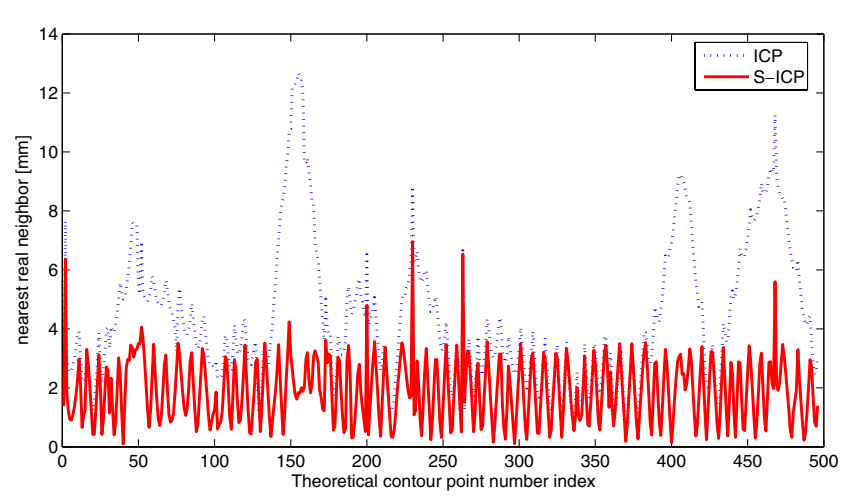

Fig. 10 Registration error along the contour

$1.9 \mathrm{~mm}$ when the distance between points is $2 \mathrm{~mm}$ for the theoretical contour, i.e. the mean error has been reduced from $57 \%$ and the peak error from $45 \%$ with respect to rigid ICP. Computing time is below $1 \mathrm{~s}$ for the porthole example (around 1,500 points) and is $26 \mathrm{~s}$ for the nose-cone (around 10,000 points) on a PC equipped with a Dual core processor $2,16 \mathrm{GHz}$ and $1 \mathrm{G} \mathrm{Ram}$, which is in accordance with a tuning procedure schedule within production context of small series. Note that results in Fig. 10 correspond to a point cloud (object) with measurement noise and missing points. A residual random noise can indeed be noticed when using the S-ICP method, whereas a basic ICP method suffers from matching errors depending from contour curvature (which can be seen when analyzing residuals). Of course, other methods, including nonrigid ICP methods, can lead to similar results, but at the

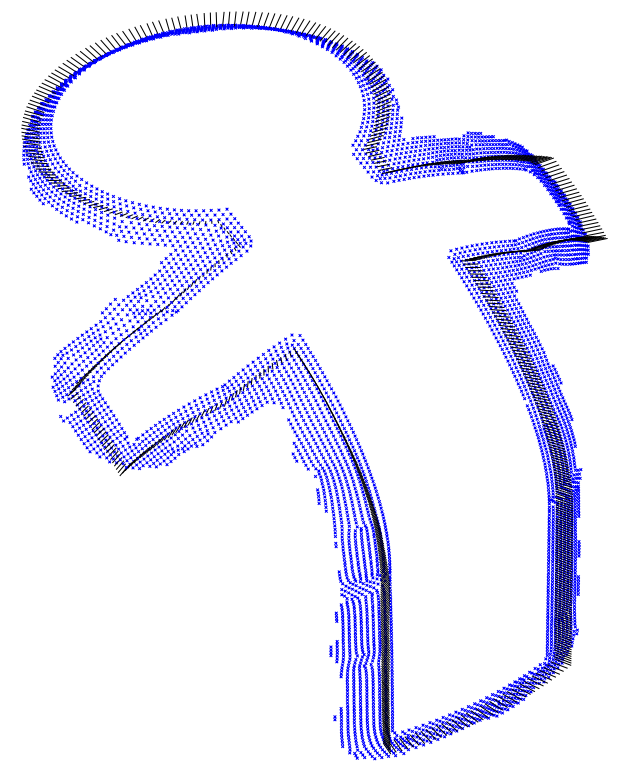

Fig. 11 Trajectory update and tool normals after S-ICP
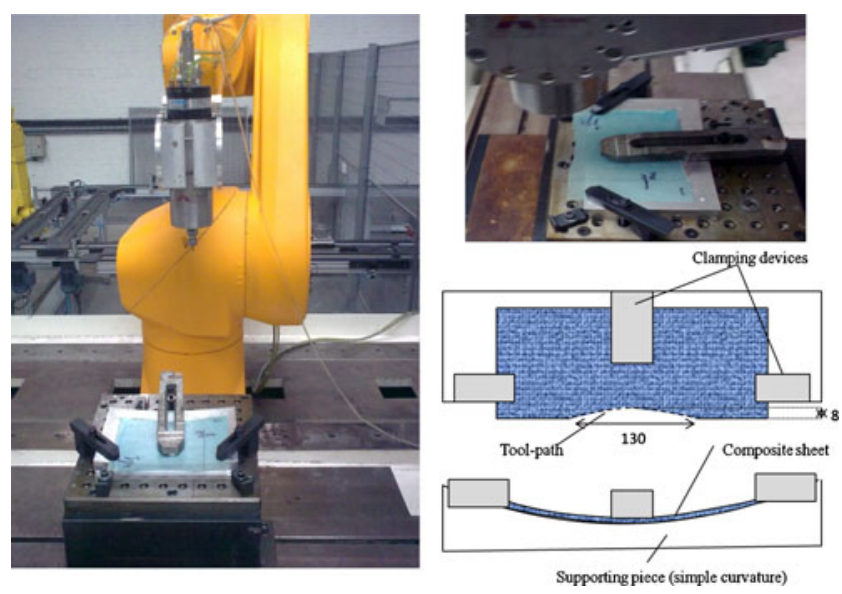

Fig. 12 Robotic Milling work cell and experimental setup

expense of more complicated and lengthy computations or tuning procedures.

Moreover, the resulting transformation can be applied to the deburring of the workpiece; Fig. 11 shows the updated tool trajectory designed to machine the actual workpiece. The robot trajectory is updated as follows:

a. For every trajectory point, find the nearest neighbor in the set of model points

b. Apply the S-ICP transform to the point if the neighboring point belongs to a region with no overlap, otherwise, apply the transforms for both segments and use the overlapping weighted procedure.

c. The normals to the tools are updated using the new trajectory points.

\subsection{Experimental results on a simple test case}

In the present work, a robotic milling work cell is setup with a Staubli RX170 industrial manipulator depicted in Fig. 12. A high-speed spindle $(30,000 \mathrm{tr} / \mathrm{min})$

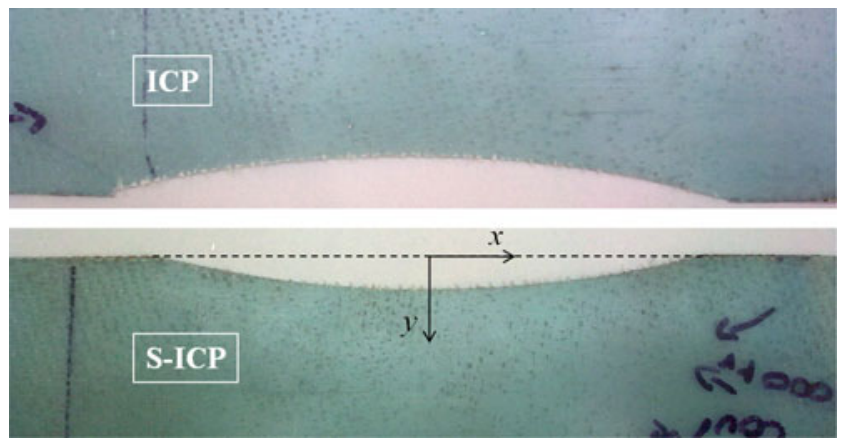

Fig. 13 Finished parts 


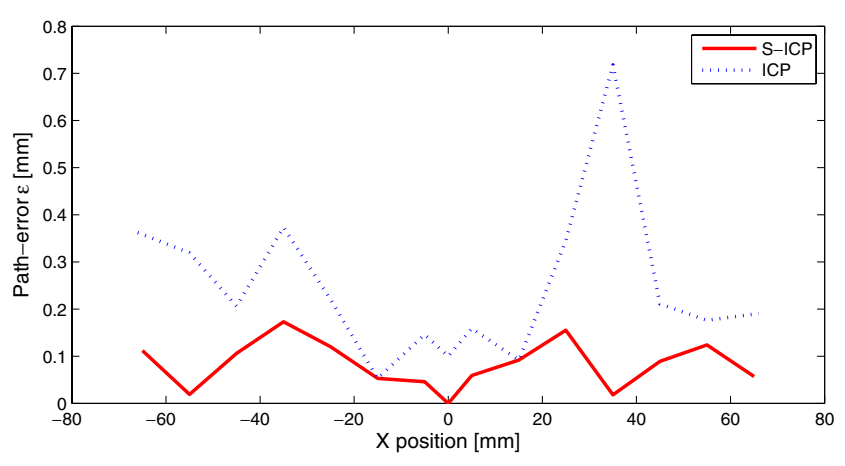

Fig. 14 Path-error measurements (absolute abscissa error according to the theoretical contour) a performing method has been found that it has the advantage of being model-free. Experimental validations, using a machining 6-axis robot, have demonstrated the effectiveness of the proposed approach. Hence, trajectory update can be directly derived, allowing on-site machining by robot without accurate clamping or reengineering design.

Acknowledgements The Authors wish to thank Mr. Xu Z. for programming the algorithm and Mr. Helle X. and Mr. Olabi A. for their contributions to the experimental validations.

\section{References}

is mounted on the robot wrist while the workpiece is fixed on a steel table. A simple test case is presented which consists in deburring a circular contour on a constrained composite material. The programmed toolpath assumes that the unconstrained composite sheet is flat. The real deformation of the sheet is imposed by the supporting piece, and hence is known. Figure 12 shows the experimental setup. The sheet deformation was measured using a 3D scanner device (HandyscanTM) in the machining region (about 3,000 points). After completing the machining operation, the composite sheet is unclamped and the performance criterion is chosen as the absolute abscissa error according to the theoretical contour, noted $\varepsilon$. Figure 13 presents a photo of the finished part and the measured path error is represented in Fig. 14. A better circularity of the machining part after the S-ICP registration method can be clearly observed. The residual error after S-ICP (around $0.2 \mathrm{~mm}$ ) is in the range of the industrial robot accuracy required for deburring operations. Hence, this simple test case demonstrates the effectiveness of the proposed algorithm.

\section{Conclusion}

A new registration algorithm has been applied to update the tool-path for deburring operations on deformed workpieces, which uses techniques derived from the Iterative Closest Point method. After a first coarse ICP, the deformation was modelled in a neighborhood of the contour to be deburred. This technique is only suitable for very simple shapes and is not realistic for registration during production of such complex parts as aircraft nose-cones. Using contour subdivision,
1. Amberg B, Romdhani S, Vetter T (2007) Optimal step nonrigid icp algorithms for surface registration. In: IEEE conf. computer vision and pattern recognition, CVPR '07

2. Besl PJ, McKay ND (1992) A method for registration of 3-D shapes. IEEE Trans Pattern Anal Mach Intell 14:239-256

3. Ben Amor B, Ardabilian M, Chen L (2006) New experiments on ICP-Based 3D face recognition and authentication. In: ICPR '06: proceedings of the 18th international conference on pattern recognition, Hong Kong, pp 1195-1199

4. Boukebbab S, Bouchenitfa $\mathrm{H}$, Boughouas $\mathrm{H}$, Linares JM (2007) Applied iterative closest point algorithm to automated inspection of gear box tooth. Comput Ind Eng 52:162-173

5. Chen Y, Medioni G (1992) Object modelling by registration of multiple range images. Image Vis Comput 10:145-155

6. Chuang CM, Chen CY, Yau HT (2002) A reverse engineering approach to generating interference-free tool paths in threeaxis machining from scanned data of physical models. Int $\mathrm{J}$ Adv Manuf Technol 19:23-31

7. Ezra E, Sharira M, Efrat A (2008) On the performance of the ICP algorithm. Comput Geom 41:77-93

8. Horn BKP (1987) Closed-form solution of absolute orientation using unit quaternions. J Opt Soc Am A 4:629-642

9. Jost T, Hügli H (2002) Fast ICP algorithms for shape registration, pattern recognition. In: 24th DAGM symposium proceedings, pp 91-99

10. Kambhamettu C, Goldgof D, He M, Laskov P (2003) 3D nonrigid motion analysis under small deformations. Image Vis Comput 21:229-245

11. Kim D, Kim D (2010) A fast ICP algorithm for 3-D human body motion tracking. IEEE Signal Process Lett 17:402-405

12. Laskov P, Kambhamettu C (2003) Curvature-based algorithms for nonrigid motion and correspondence estimation. IEEE Trans Pattern Anal Mach Intell 25:1349-1354

13. Li M, Kambhamettu C, Stone M (2007) Nonrigid motion recovery for 3D surfaces. Image Vis Comput 25:250-261

14. Liu Y (2004) Improving ICP with easy implementation for free-form surface matching. Pattern Recogn 37:211-226

15. Okada N, Hebert M (2003) Fast 3D tracking of non-rigid objects. In: IEEE ICRA 2003, pp 3497-3503

16. Pahk HJ, Ahn WJ (1996) Precision inspection system for aircraft parts having very thin features based on CAD/CAI integration. Int J Adv Manuf Technol 12:442-449

17. Pottmann H, Leopoldseder S, Hofer M (2004) Registration without ICP. Comput Vis Image Underst 95:54-71 
18. Ravishankar S, Dutt HNV, Gurumoorthy B (2010) Automated inspection of aircraft parts using a modified ICP algorithm.

19. Saadat M, Cretin L, Sim R, Najafi F (2009) Deformation analysis of large aerospace components during assembly. Int J Adv Manuf Technol 41:145-155

20. Seltzer M, Von Stryk O, Abele E, Bauer J, Weigold M (2008) High speed cutting with industrial robots: towards model based compensation of deviations. In: Proceedings of Robotik 2008, 11-12 June, Munich, Germany, pp 143-146

21. Tsai MJ, Huang JF, Kao WL (2009) Robotic polishing of precision molds with uniform material removal control. Int J Mach Tools Manuf 49:885-895

22. Zhu L, Barhak J, Srivatsan V, Katz R (2007) Efficient registration for precision inspection of free-form surfaces. Int $\mathrm{J}$ Adv Manuf Technol 32:505-515 